IAU Colloquium 190 on Magnetic Cataclysmic Variables

ASP Conference Series, Vol. 315, 2004

Sonja Vrielmann \& Mark Cropper, eds.

\title{
Modeling the radio outbursts in AE Aquarii
}

\author{
L. A. Venter and P. J. Meintjies \\ Physics Department and UFS Boyden Observatory, PO Box 339, \\ University of the Free State, Bloemfontein, 9300, South Africa
}

\begin{abstract}
In this paper we model the non-thermal radio to infra-red flares from AE Aqr. In our model the non-thermal flares originate in highly magnetized $\left(B_{b l o b} \geq 2000 \mathrm{G}\right)$ blobs that may be among the propeller ejected outflow. It was shown that the condition $\beta \leq 1$ constrains the frozen-in magnetic field in these blobs to $\mathrm{B}_{b l o b} \geq 2000 \mathrm{G}$, which is of the same order of magnitude as the inferred polar field of the secondary. As these magnetized blobs encounter the violent mhd-propeller, processes such as reconnection, magnetic pumping, and shocks will result in continuous acceleration of electrons from $(\gamma=2 \rightarrow 30 ; \delta=2.8 \rightarrow 2.6)$ with resultant synchrotron emission. The total radio to infra-red flare spectrum was modelled in terms of such expanding magnetized synchrotron emitting blobs in various stages of their evolution from $\rho=\left(\mathrm{r} / \mathrm{r}_{\circ}\right)=1 \rightarrow$ 400. In terms of our model, the total integrated flux during outbursts, over the wide frequency range from $1 \mathrm{GHz}$ is the result of several $(\sim 20)$ synchrotron emitting blobs observed in different stages of their evolution, resulting in a spectrum showing a peak flux of $\mathrm{S}_{\nu} \sim 148 \mathrm{mJy}$ at $\nu \sim$ $1805 \mathrm{GHz}$ ( $\sim 166$ microns), where the spectrum changes from a typical self-absorbed $\mathrm{S}_{\nu} \propto \nu^{\alpha}$ spectrum to $\mathrm{S}_{\nu} \propto \nu^{-(\delta-1) / 2}$ spectrum, i.e. where the blobs are combined optically thin.
\end{abstract}

\section{The non-thermal radio properties of AE Aqr}

The first manifestation of the non-thermal properties of this system was the discovery of variable non-thermal radio emission (Bookbinder \& Lamb 1987) at $\nu=1.4 \mathrm{GHz}$ and $\nu=5 \mathrm{GHz}$ respectively. Immediately after this first campaign, AE Aqr was observed again with the VLA at $1.5 \mathrm{GHz}, 4.9 \mathrm{GHz}, 15 \mathrm{GHz}$ and $22.5 \mathrm{GHz}$, revealing nearly continuous radio synchrotron outbursts superimposed on a weak quiescent background emission (Bastian, Dulk \& Chanmugam 1988), mimicking Cyg X-3 on a lower scale. This variable radio emission was interpreted as non-thermal $\left(10^{9} \mathrm{~K}<\mathrm{T}_{B}<10^{12} \mathrm{~K}\right)$ incoherent flares involving relativistic electrons with energies $\gamma \approx 3-30$ (Bastian, Dulk \& Chanmugam 1988). The general time-averaged shape of the radio spectrum up to $15 \mathrm{GHz}$ resembled a self-absorbed power law $S_{\nu} \sim S_{\circ} \nu^{\alpha}$ with an index $\alpha=0.3-0.4$ (Bastian, Dulk \& Chanmugam 1988). More recent radio (Abada-Simon et al. 1993, 1995a,b), and infra-red (IR) observations, using ISOPHOT-C, (Abada-Simon et al. 1999,2002 ) at $\nu \sim 3333 \mathrm{GHz}$ (90 microns) confirmed that the self-absorbed spectrum possibly extends up to $\nu \sim 3333 \mathrm{GHz}$ (90 microns). The detection at 90 microns corresponds to a measured flux level (at 5 sigma level) of $S_{\nu} \sim$ $113 \mathrm{mJy}$ (Abada-Simon et al. 2002). It is anticipated that the detection of the 
90 micron emission must be very close to the turning point of the self-absorbed spectrum, since Abada-Simon et al. (2002) estimated IRAS upper limits of $34 \mathrm{mJy}, 51 \mathrm{mJy}$ and $73 \mathrm{mJy}$ at 12,25 and $60 \mu \mathrm{m}$ respectively (i.e. 25000 $\mathrm{GHz}, 11538 \mathrm{GHz}$ and $5000 \mathrm{GHz}$ ). The time averaged spectrum up to $3333 \mathrm{GHz}$ (90 microns) can be approximated as $S_{\nu}=\mathrm{S}_{1}\left(\frac{\nu}{1 G H z}\right)^{0.5}$, with $\mathrm{S}_{1} \approx 3.15 \mathrm{mJy}$ (Abada-Simon et al. 1999, 2002). The maximum observed non-thermal flux, $\mathrm{S}_{\max } \sim 113\left(\frac{\nu_{\max }}{3333 \mathrm{GHz}}\right)^{0.5} \mathrm{mJy}$, results in a maximum non-thermal radio to IR luminosity of

$$
L_{R-I R} \approx 5 \times 10^{30}\left(\frac{\Omega}{4 \pi}\right)\left(\frac{S_{\circ}}{113 \mathrm{mJy}}\right)\left(\frac{D}{100 \mathrm{pc}}\right)^{2} \mathrm{erg} \mathrm{s}^{-1} .
$$

several orders of magnitude lower than the spin-down power of the magnetized white dwarf, i.e.

$$
\begin{aligned}
\beta & \sim\left(\frac{L_{R-I R}}{I \Omega \dot{\Omega}}\right) \\
& \sim 5 \times 10^{-4}\left(\frac{I \Omega \dot{\Omega}}{10^{34} \mathrm{erg} \cdot \mathrm{s}^{-1}}\right)^{-1}\left(\frac{L_{R-I R}}{5 \times 10^{30} \mathrm{erg} \cdot \mathrm{s}^{-1}}\right),
\end{aligned}
$$

implying that only a small fraction of the spin-down power is converted to nonthermal radio synchrotron emission.

\section{Magnetized fragmented mass transfer}

It has been shown (this proceedings) that the secondary star in $\mathrm{AE} A q r$ has a surface polar field strength of $B_{\circ} \approx 2000 \mathrm{G}$. The $\alpha^{2}$-dynamo models for magnetic field generation in convective stars predict that star spots and prominences exist in the equatorial plane of convective secondary stars in binary systems (Hessman 2003, personal communication). These magnetic structures are regions of high field strength and if such structures extend far enough from the surface of the mass transferring star, they may be gravitationally forced to pinch off and fall across into the Roche lobe of the white dwarf. Alternatively, magnetic flux may also be frozen in the mass transfer flow that leaves the L1 region, which may result in a fraction of the mass transferred from the secondary star to the white dwarf consisting of magnetized blobs. Turbulent fluid motions inside the blobs may also result in the field being contorted and twisted, resulting in localized magnetic knots inside the blobs (Spruit 2002, personal communication). In these regions the blob may be magnetically dominated, i.e. the ratio $\beta=$ $\left(\mathrm{P}_{\text {gas }} / \mathrm{P}_{\text {mag }}\right.$ ) $\leq 1$ (where $\mathrm{P}_{\text {gas }}=\mathrm{nk}_{B} \mathrm{~T}$ is the gas pressure and $\mathrm{P}_{m a g} \mathrm{~B}^{2} / 8 \pi$ is the magnetic pressure). The minimum magnetic field for a $\beta \leq 1$ plasma can be obtained from equipartition between gas and magnetic pressure in the blob. There is observational evidence from Ginga data that the accretion flow may be clumpy, with particle densities between $N_{p} \approx 10^{15}-10^{17} \mathrm{~cm}^{-3}$ (Beardmore \& Osborne 1997; Meintjes 2002), with typical temperatures of the order of $\mathrm{T}_{b} \approx$ $10^{4}-10^{5} \mathrm{~K}$ (Eracleous \& Horne 1996). Therefore, the minimum field strengths for a $\beta \leq 1$ fluid inside blobs which leave the L1 region (with sizes comparable to the white dwarf radius $r_{0} \approx 10^{9} \mathrm{~cm}$ ) are of the order of

$$
B_{e q} \geq 2300\left(\frac{N_{\circ}}{10^{16} \mathrm{~cm}^{-3}}\right)^{1 / 2}\left(\frac{T_{b}}{10^{5} \mathrm{~K}}\right)^{1 / 2}\left(\frac{\rho}{\rho_{i}}\right)^{-3 / 2} \mathrm{G} \text {. }
$$


The particle density in these expanding blobs is assumed to scale as $N_{p}=N_{\circ} \rho^{-3}$, where $\rho=\left(r / r_{0}\right)$ represents the blob size at any given instant, resulting in the scaling of the field according to $\mathrm{B} \geq \mathrm{B}_{\circ} \rho^{-3 / 2}$.

The severe interaction between the fast rotating magnetic propeller and the blobs may result in a small fraction of the spin-down power of the white dwarf being pumped into particle acceleration in dense magnetized blobs, with resulting radio emission through the van der Laan process as these blobs expand. To order of magnitude, one can estimate the energy pumped into expanding blobs as the white dwarf magnetic field sweeps over and compresses them. The MHD power pumped into expanding blobs by the rotating magnetic field of the white dwarf is

$$
\begin{aligned}
L_{b l o b} & \approx\left(\frac{B^{2}(r)}{8 \pi}\right)\left(\frac{4 \pi}{3}\right) R_{b}^{3} \Omega_{*} \\
& \leq 10^{32}\left(\frac{B_{*}}{2 \times 10^{6} G}\right)^{2}\left(\frac{P_{w d}}{33 s}\right)^{-1}\left(\frac{R_{w d}}{10^{9} \mathrm{~cm}}\right)^{3} \text { erg.s } \mathrm{s}^{-1}
\end{aligned}
$$

This power is sufficient to excite processes resulting in particle acceleration and non-thermal radio emission from expanding blobs, given that the maximum radio luminosity from AE Aqr, derived from the Abada-Simon et al. (1999, 2002) spectrum, up to $3333 \mathrm{GHz}$ is approximately $L_{R-I R} \leq 10^{31} \mathrm{erg} \cdot \mathrm{s}^{-1}$. These processes will be the focus of the rest of the paper.

\section{Particle acceleration}

We argue that magnetic reconnection through the fast Petschek (1964) mechanism can accelerate particles in a blob with a field of $\mathrm{B}_{e q} \approx 2300 \mathrm{G}$, to energies of the order of

$$
\varepsilon_{e} \leq 1.6\left(\frac{B_{e q}}{2300 G}\right)\left(\frac{T_{b}}{10^{5} K}\right)^{-1 / 2}\left(\frac{l_{r e c}}{10^{-2} r_{\circ}}\right)^{-1}\left(\frac{l_{r e c}}{10^{-2} r_{\circ}}\right) \mathrm{MeV}
$$

Initially, in a highly magnetized $\mathrm{B}_{b, i} \approx 2000 \mathrm{G}$, dense $\mathrm{N}_{p, i} \approx 10^{16} \mathrm{~cm}^{-3}$ plasma, synchrotron losses of mildly relativistic electrons will be the dominant energy loss mechanism. For sufficient acceleration initially, reconnection must occur on a shorter timescale than the synchrotron loss timescale for $\varepsilon_{e} \sim 1 \mathrm{MeV}$ electrons, which is

$$
t_{s, i} \approx 60\left(\frac{\gamma}{2}\right)^{-1}\left(\frac{B}{2300 \mathrm{G}}\right)^{-2} \mathrm{~s} .
$$

Reconnection can typically occur on timescales $\tau_{\text {rec }} \approx\left(l_{\text {rec }} / \mathrm{v}_{i}\right)$ where $l_{\text {rec }}$ and $\mathrm{v}_{i}$ represent the length scale of magnetic reconnection and speed at which the field lines approach one another, typically a fraction $(\eta \sim 0.1)$ of the Alfvén speed $\left(\mathrm{v}_{A}=(\mathrm{B} / \sqrt{4 \pi \rho})\right.$, i.e. $\mathrm{v}_{i} \approx \eta(\mathrm{B} / \sqrt{4 \pi \rho})$ (Parker 1976). Since we consider $l_{\text {rec }}$ $\approx 10^{-2} \mathrm{r}_{\mathrm{o}}$, we can see that the reconnection and hence acceleration timescale is approximately

$$
\tau_{r e c} \approx 20\left(\frac{l_{r e c}}{10^{-2} r_{\circ}}\right)\left(\frac{v_{i}}{0.1 v_{A}}\right)^{-1} \mathrm{~s}
$$

which is significantly shorter than the synchrotron loss timescale. 
As the battered magnetic blobs are heated and expand, expansion losses $\left(\mathrm{t}_{\exp } \sim \mathrm{r} / \mathrm{v}_{\text {exp }}\right)$ take over from synchrotron losses as the dominant energy loss mechanism, i.e.

$$
\begin{aligned}
& t_{s} \approx 300\left(\frac{\gamma}{20}\right)^{-1}\left(\frac{B}{100 \mathrm{G}}\right)^{-2} \mathrm{~s} \\
& t_{\text {exp }} \approx 180\left(\frac{r(t)}{4 a}\right)\left(\frac{v_{\text {exp }}}{0.01 c}\right)^{-1} \mathrm{~s}
\end{aligned}
$$

where $t_{\text {exp }}$ corresponds to a VLBI radio measurement (A.E. Niell, personal communication) of a typical expanding synchrotron emitting cloud, expanding with a speed $\mathrm{v}_{\text {exp }} \sim 0.01 \mathrm{c}$ until it reaches a size of approximately $\rho=\left(\mathrm{r} / \mathrm{r}_{\mathrm{o}}\right) \rightarrow 400$, corresponding to a size of approximately 4 times the binary separation. These relatively slow losses during the expansion phase of the blobs may be instrumental in the net gain of electron energies through processes such as magnetic pumping and shock drift acceleration (fast-Fermi process).

As these blobs are propelled out of the system, magnetic compression and blobs ramming into one another may result in strong oblique shocks as well as magnetic instabilities generated in the magnetized blobs. It can be shown that the fast-Fermi and magnetic pumping mechanisms respectively can result in a relative quick energizing of an electron population in regions where $\beta<1$. For example, the energy increase per encounter (Toptyghin 1980), is

$$
\begin{gathered}
\frac{\Delta E_{\max }}{E_{i}}=\left(4\left(\frac{B_{d}}{B_{u}}\right)-3\right) \text { (thermal) } \\
\frac{\Delta E_{\max }}{E_{i}}=\left(2\left(\frac{B_{d}}{B_{u}}\right)-1\right) \text { (relativistic) }
\end{gathered}
$$

for shock drift acceleration, and

$$
\left(\frac{E(t)}{E_{\circ}}\right)=\left(\frac{B_{w}}{B_{u}}\right) \sin ^{2} \theta_{\circ}\left(t_{\circ}\right)
$$

for magnetic pumping (Parker 1976). Since for strong shocks $\left(\mathrm{B}_{d} / \mathrm{B}_{u}\right) \rightarrow 4$, both these mechanisms can result in a relatively rapid energizing of a mildly relativistic $(\sim 1 \mathrm{MeV})$ electron population if the electron losses are relatively slow. For example, shock drift acceleration boosts the energy of thermal and mildly relativistic electrons by factors of $\left(\Delta \mathrm{E}_{\max } / \mathrm{E}_{i}\right) \rightarrow 13$ and $\left(\Delta \mathrm{E}_{\max } / \mathrm{E}_{i}\right) \rightarrow 7$ respectively in a single encounter with a strong shock. For continued acceleration the particle population may be sufficiently heated to mildly relativistic energies of $\gamma \leq 30(\rho \rightarrow 400)$ as the blobs expand, with a resultant slight hardening of the spectrum from $\delta \approx 2.8 \rightarrow 2.6$.

\section{The van der Laan (VDL) model}

The model (van der Laan 1966) incorporates a power law electron energy distribution $n(E) d E=K E^{-\delta} d E$ with $\mathrm{K}$ related to $\mathrm{N}$ (number density of electrons with $\left.E>E_{c}\right)$, as $K=(\delta-1) E_{c}^{\delta-1} N$. A single flare is taken as a blob of relativistic electrons with a magnetic field frozen into the plasma. Bastian, Dulk \& 
Chanmugam (1988) give a parameterization for a single blob or flare according to the VDL model as

$$
\begin{aligned}
S(\nu, \rho)= & S_{m \circ}\left(\frac{\nu}{\nu_{m \circ}}\right)^{5 / 2} \rho^{3} \\
& \times \frac{\left\{1-\exp \left[-\tau_{m}\left(\nu / \nu_{m \circ}\right)^{-(\delta+4) / 2} \rho^{-(2 \delta+3)]\}}\right.\right.}{1-\exp \left(-\tau_{m}\right)}
\end{aligned}
$$

where $\mathbf{S}_{m \circ}$ is the maximum flux density at initial time $t_{\circ}, \nu_{m \circ}$ is the frequency at this maximum and $\tau_{m}$ is the optical depth at this frequency. The maximum flux density (zero expansion, $\rho=1$ ) can be estimated from Dulk (1985), which is summarized in Bastian, Dulk \& Chanmugam (1988). For single flare events we have

$$
\begin{aligned}
S_{m, \circ}= & A_{1}\left[A_{2} \frac{\delta-1}{\delta+2}\left(\frac{E_{c}}{1 \mathrm{MeV}}\right)^{\delta-1} N_{\circ}\right]^{3 /(2 \delta+3)} \\
& \times\left[r_{\circ}^{(4 \delta+9) / 3} B_{\circ}^{(\delta+3) / 6}\right]^{3 /(2 \delta+3)} \nu_{m}{ }^{(7 \delta+3) /(4 \delta+6)}
\end{aligned}
$$

with $A_{1}=3.3 \times 10^{6} \times 2^{-\delta / 2} \pi \mathrm{k} / c^{2} D^{2}, A_{2}=\left(2.8 \times 10^{7}\right)^{(\delta+4) / 2} / 10^{11}$ and

$$
N_{\circ}=A_{3}\left[\frac{\delta+2}{\delta-1}\left(\frac{E_{c}}{1 \mathrm{MeV}}\right)^{-(\delta-1)}\left(\frac{B_{\circ}^{2 \delta+9}}{r_{\circ}}\right)\right]^{1 /(\delta+5)},
$$

where $N_{\circ}$ represents the electron number density assuming equipartition between the magnetic and relativistic electron energy densities. In this equation the constant $A_{3}=\left[3.2 \times 10^{4} \times 2^{\delta / 2}\left(10^{11}\right)^{1 /(\delta+4)}\right]^{(\delta+4) /(\delta+5)}$. By substituting Equations 14 and 15 into Equation 13, the expected flux densities of individual flare events can be simulated.

\section{Modeling the radio outbursts}

It can be shown (e.g. Pacholzyk 1970) that the total energy in relativistic particles in a synchrotron source is

$$
E_{e}(\mathrm{erg})=\frac{L\left(\mathrm{erg} \cdot \mathrm{s}^{-1}\right)}{2 \times 10^{-3}}\left(\frac{3-\delta}{2-\delta}\right) c_{1}^{1 / 2} B^{-3 / 2}\left[\frac{\nu_{0}^{\frac{2-\delta}{2}}-\nu^{\frac{2-\delta}{2}}}{\nu_{0}^{\frac{3-\delta}{2}}-\nu^{\frac{3-\delta}{2}}}\right] .
$$

The total magnetic energy in the source is $E_{B}(\operatorname{erg})=\frac{B^{2} R^{3} \Phi}{6}$ with $\Phi$ the fraction of volume occupied by the field and relativistic particles (e.g. Pacholzyk 1970). Then ignoring particles other than electrons it can be shown (e.g. Pacholzyk 1970) that equipartition between the magnetic field and the particle energy, minimizing the energy, gives

$$
B_{\text {min }}=\left[\frac{3}{4} \frac{C_{e}(\delta)}{8 \pi} \frac{L\left(\text { erg.s } \mathrm{s}^{-1}\right)}{V_{\text {source }}}\right]^{2 / 7} .
$$

From the observed spectrum of AE Aqr (Abada-Simon et al. 1999, 2002) the maximum synchrotron luminosity of $\mathrm{AE} A \mathrm{Aqr}$ was estimated to be of the order of $\mathrm{L}_{R-I R} \sim 5 \times 10^{30}$ erg.s ${ }^{-1}$. It is easy to show that for initial blob radii between $\mathbf{r}_{\circ}$ $\approx 10^{8}-10^{9} \mathrm{~cm}$, equipartition magnetic fields in the blobs of the order of $\mathrm{B}_{b l o b} \geq$ 

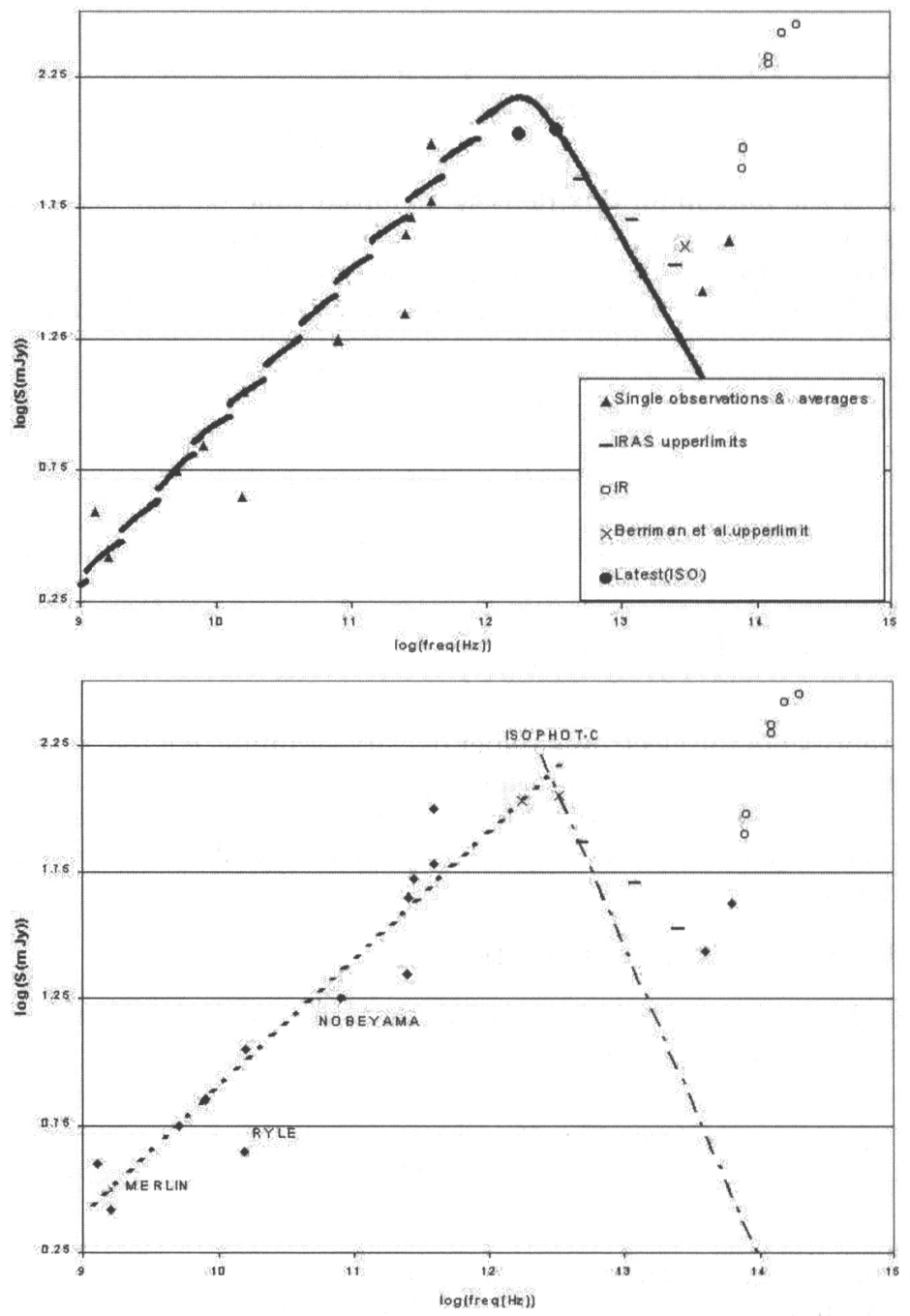

Figure 1. The simulated radio to IR spectrum representing the total integrated flux from approximately 20 , initially highly magnetic, synchrotron emitting blobs in different stages of their evolution. The second spectrum shown in this figure represents a fit to the radio measurements, adapted form Abada-Simon et al. (1999).

$650-2 \times 10^{4} \mathrm{G}$ are implied. This confirms our previous estimate that the magnetic field strength in the blobs can be large. The spectrum was simulated using a total of $\approx 20$ expanding synchrotron emitting blobs in different stages 
of their evolution, from $\rho=(1-400)$ and is represented in Figure 1 (top panel), corresponding well with the spectrum inferred from observations (bottom panel).

\section{Discussion}

In this paper a model is proposed to explain the observed non-thermal radio outbursts of AE Aqr in terms of expanding radio emitting magnetized blobs. It is proposed that the observed $\mathrm{S}_{\nu} \propto \mathrm{v}^{\alpha}$ power law spectrum can be explained in terms of radio synchrotron emission from expanding, strongly magnetized $(\beta \leq 1)$ blobs, in different stages of their evolution. In terms of our model, the simulated radio - infra-red spectrum is a superposition of several $(\leq 20)$ synchrotron emitting bubbles (van der Laan mechanism) in different stages of expansion from $\rho=\left(\mathrm{r} / \mathrm{r}_{0}\right)=1-400$, containing electron populations with energies between $\gamma \approx 2(\rho=1 ; \delta=2.8)$ and $\gamma \approx 30(\rho \approx 400 ; \delta=2.6)$.

Acknowledgments LAV acknowledges partial financial support received from IAU to take part in the IAU Colloquium on MCVs.

\section{References}

Abada-Simon M., Lecacheux A., Bookbinder J. \& Dulk G.A., 1993, ApJ, 406, 692

Abada-Simon M., Bastian T.S., Horne K. \& Bookbinder J.A., 1995a, in Buckley D.A.H., Warner B., eds, ASP conf. Ser. Vol 85, Proc. Cape Workshop on Magnetic Cataclysmic Variables, p. 355

Abada-Simon M., Bastian T.S., Bookbinder A.J., Aubier M., Bromage G., Dulk G.A. \& Lecacheux A., 1995b, in Lecture Notes in Physics, 454, 268

Abada-Simon M., Mouchet M., Aubier M., Barrett P., de Jager O.C., de Martino D. \& Ramsay G., 1999, in Cox, P. Kessler, M.F. eds., ESA SP-427, Proc. The Universe as seen by ISO. ESA, Paris, p.257

Abada-Simon, M., Mouchet, M., Casares, J. (+13 co-authors), 2002, in Combes F., Barret D. eds., Semaine de l' Astrophysique Francaise, EdP-Sciences Bastian T.S., Dulk G.A. \& Chanmugam G., 1988, ApJ, 324, 431 (BDC)

Beardmore A.P. \& Osborne J.P., 1997, MNRAS, 290, 145

Bookbinder J.A. \& Lamb D.Q., 1987, ApJ, 323, L131

Dulk G.A., 1985, ARA\&A, 23, 169

Eracleous M. \& Horne K., 1996, ApJ, 471, 427

Meintjes, P.J. 2002, MNRAS, 336, 265

Pacholzyk A.G. 1970, Radio Astrophysics, W.H. Freeman \& Co. San Francisco

Parker E.N. 1976, The physics of non-thermal radio sources, G. Seti (ed.), D. Reidel Publishing company, Dordrecht-Holland, p. 137

Petschek H.E., 1964, AAS-NASA Symposium on the physics of solar flares, NASA Special publications SP-50, p.425

Toptyghin I.N., 1980, Space Sci.Rev.., 26, 157

van der Laan, H., 1966, Nature, 211, 1131 